\title{
Pseudoaneurysm of peroneal artery after ankle arthroscopy
}

\author{
Daniela Battisti ${ }^{1}$ \\ Francesco Oliva ${ }^{1}$ \\ Umberto Tarantino ${ }^{1}$ \\ Maffulli Nicola ${ }^{2}$
}

1 Department of Orthopaedics and Traumatology, University of Rome "Tor Vergata", "Policlinico Tor Vergata" Foundation, Rome, Italy

2 Head of Department of Physical and Rehabilitation Medicine, Azienda Ospedaliera San Giovanni di Dio e Ruggi d'Aragona, University of Salerno, Italy; Queen Mary University of London, Barts and the London School of Medicine and Dentistry, Centre for Sports and Exercise Medicine, Mile End Hospital, London, UK

Corresponding author:

Francesco Oliva

Department of Orthopaedics and Traumatology, University of Rome "Tor Vergata",

"Policlinico Tor Vergata" Foundation

V.le Oxford, 81

00133 Rome, Italy

E-mail: olivafrancesco@hotmail.com

\section{Summary}

Background: ankle arthroscopy increased its role in the diagnosis and treatment of pathology of the ankle since 1970s. Although the benefits are well established, ankle arthroscopy is associated with a definite risk of complications, especially neurological. The incidence rate of vascular complication after anterior ankle arthroscopy has been reported.

Methods: we review the literature on vascular complications after anterior ankle arthroscopy especially pseudoaneurysm and a case report of a peronal artery pseudoaneurysm was reported.

Conclusion: vascular complications after an anterior ankle arthroscopy are extremely rare but orthopaedic surgeons should be wary of this chance. Among vascular complications after an anterior ankle arthroscopy, peroneal artery pseudoaneurysm to our knowledge never has been described until now.
KEY WORDS: ankle arthroscopy, peroneal artery, pseudoaneurysm.

\section{Introduction}

Ankle arthroscopy was first described in 1939 by Takagi ${ }^{1}$ and increased its role in the diagnosis and treatment of pathology of the ankle since $1970 \mathrm{~s}^{2}$. It allows direct visualization of intracapsular structures so it can be helpful for several disorders of the ankle, such as articular injury, ankle instability ${ }^{3-4}$, softtissue injury, osteochondral lesions, unexplained pain or swelling, avoiding open ankle arthrotomy. It leads to less morbidity and rapid rehabilitation for the patient.

Although the benefits are well established, ankle arthroscopy is associated with a definite risk of complications. The majority of these are neurological complications that are related to portal placement ${ }^{5}$.

A pseudoaneurysm or false aneurysm develops after a damage, usually iatrogenic, to all the 3 layers of the artery wall. Vascular complications as formation of pseudoaneurysm after arthroscopic surgery are extremely rare and occur mostly after knee arthroscopy especially in relation with popliteal artery. Anteromedial and anterolateral portals used for ankle arthroscopy are considered relatively safe from vascular injuries. The incidence rate of vascular complication after anterior ankle arthroscopy is of $0,008 \%{ }^{6}$ and the anterior tibial artery is the most common vessel involved. We review the literature on vascular complication after ankle arthroscopy and here we report the case series published ${ }^{7}$. To our knowledge pseudoaneurysm of peroneal artery around the ankle has not been described after anterior ankle arthroscopy.

\section{Case report}

We describe the case of a 66-year-old Caucasian woman in good general conditions who presented to our clinic twelve weeks after a diagnostic ankle arthroscopy performed by others using standard 4.5$\mathrm{mm} 30^{\circ}$ anteromedial and anterolateral portals.

She referred swelling and superficial pain and a pulsatile mass in the external compartment of her left ankle joint. A vascular complication was clinically suspected and a Computed tomography angiography (CTA) was performed in the way to identify the mass. The CTA examination confirmed the suspected diag- 


\section{Battisti et al.}

nosis of a pseudoaneurysm arising from the peroneal artery (Fig.1).

The patient consent to be treated surgically and we performed few days later the open ligation and removal of the peroneal artery tract injured (Fig.2). Subcuticolar vycril $4 / 0$ and intradermal suture and simple medication of the skin were administered. An elastic stocking was prescribed until skin stitches were removed (15 days) ${ }^{8}$. The patient was able to return to her daily activity after two weeks without the previous discomfort. No rehabilitation protocol or drugs were used after the surgical procedure.

\section{Discussion}

A pseudoaneurysm or false aneurysm develops after a damage, usually iatrogenic, to all the 3 layers of the artery wall. It causes extravasation of blood in the surrounding tissues and the formation of fibrous tissue capsule full of blood flowing outside the injured artery. It can increase its volume because of the structure of the wall that is not made of the three layers of a natural artery and so it frequently breaks ${ }^{9}$.

The anterior tibial artery is the most common vessel involved, especially using anterocentral portal, because it runs deep to the superior and inferior retinaculum, in close proximity to the anterior ankle joint capsule. However it can be damage using anteromedial and anterolateral portals as reported in literature, during portal insertion or instrumentation through the portals or when exiting the capsule ${ }^{10}$. Also variability in the anatomic position of the vessel may contribute to the formation of pseudoaneurysms: lateral deviations are reported in $5.5 \%$ of the population and medial deviations in $3.5 \%{ }^{5-6}$.

The dorsalis pedis artery (DPA) is a continuation of the anterior tibial artery who runs until the base of
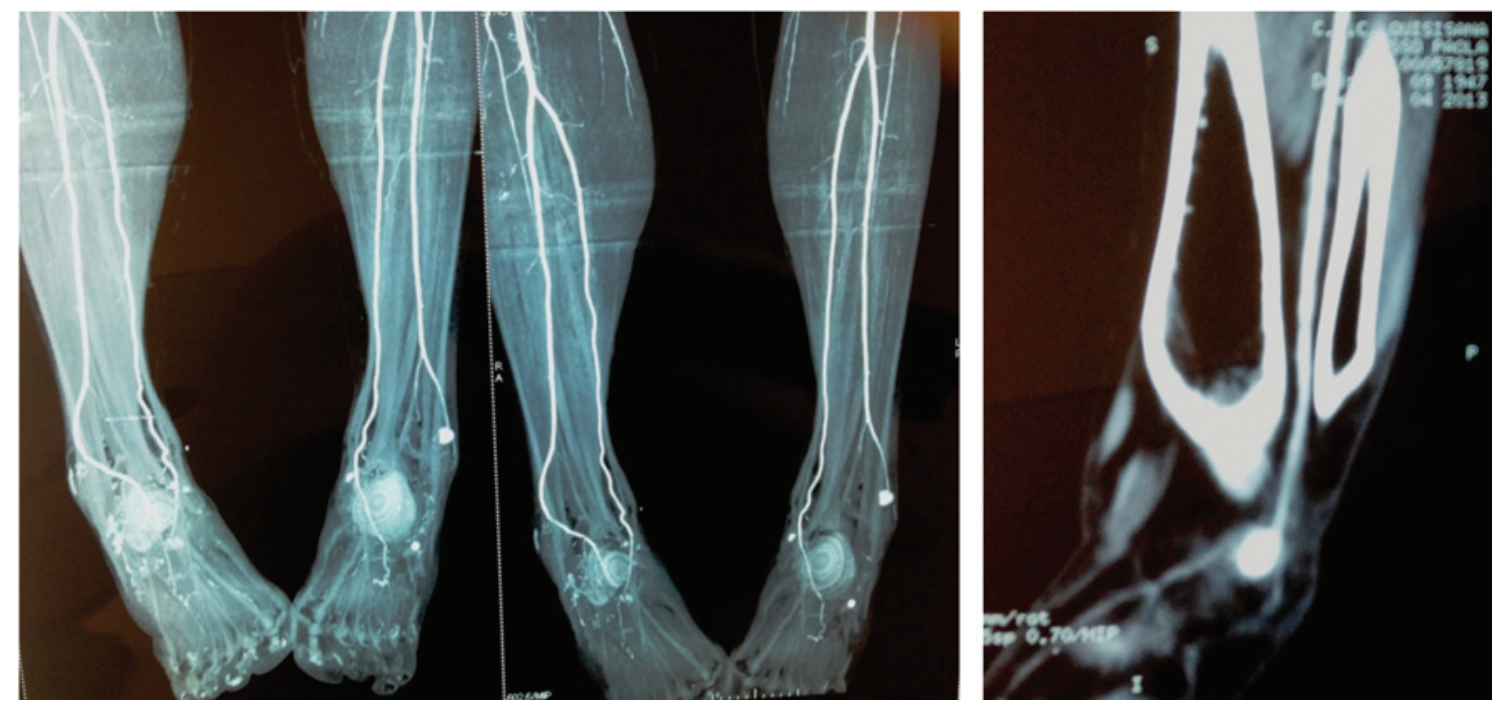

Figure 1. Computed tomography angiography (CTA) showing the formation of a pseudoaneurysm of the peroneal artery.
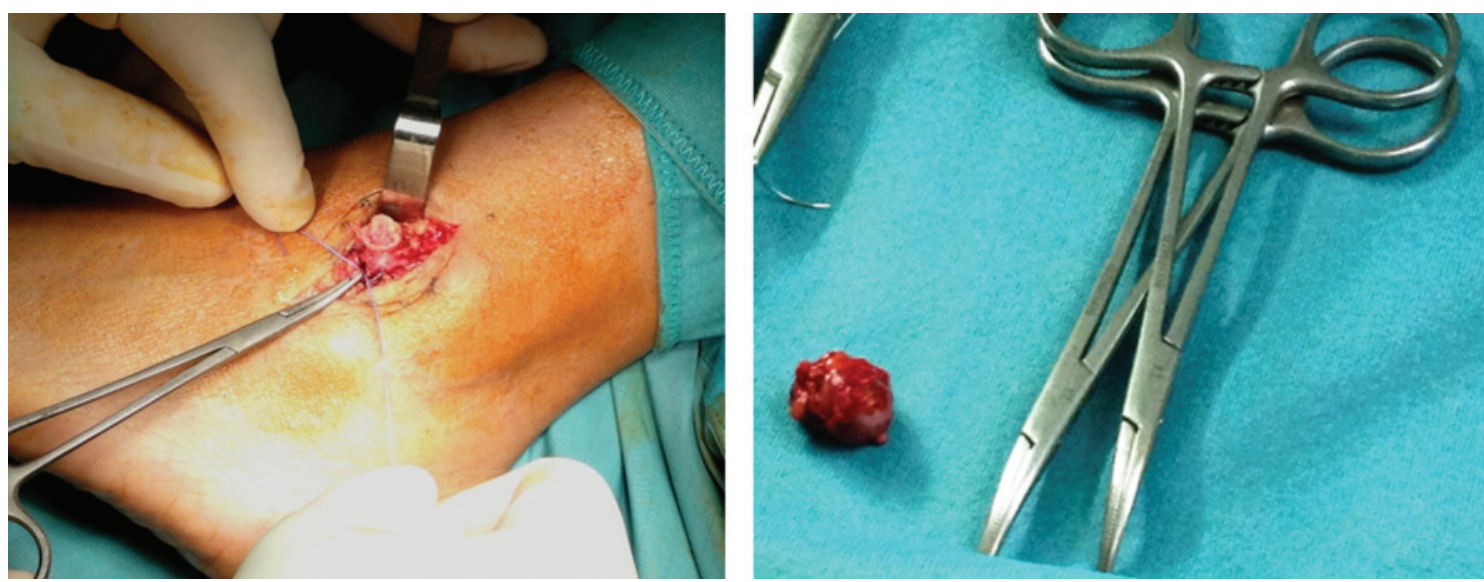

Figure 2. Intraoperative image of the pseudoaneurysm, and a comparation to a surgical instrument to show its sizes. 
first metatarsal space where joints the lateral plantar artery to form the plantar arch. The digital branch of the deep peroneal nerve runs laterally to it and medially there is the tendon of the extensor hallucis longus. The DPA is crossed by the tendon of the extensor hallucis brevis. Also anatomic variations of the DPA are described, and this can contribute to the formation of pseudoaneurysms ${ }^{11}$.

We reported for the first time in literature the formation of a pseudoaneurysm of the peroneal artery after an anterior ankle arthroscopy. The peroneal artery origins from the posterior tibial artery, $2-3 \mathrm{~cm}$ under the tendinous arch of the soleus running medially to the fibula until the tibio-tarsal joint ${ }^{12,13}$. Five cases of pseudoaneurysm of peroneal branches are described in literature after an ankle sprain?.

There are 10 cases described in literature of formation of pseudoaneurysms, in the most of them the anterior tibial artery is the vessel involved (Tab.1).

The formation of a pseudoaneurysm after an anterior ankle arthroscopy is extremely rare and usually there are no immediate signs of it. Patients may present abnormal swelling or pain of the involved ankle, with a reduction of the ROM of the joint or recurrent hemarthrosis of the ankle due to the rupture of the pseudoaneurysm. The pulsatile mass can occur after few days but can develop even after weeks or months after an anterior ankle arthroscopy and it is independent from the coagulation state of the patient ${ }^{14}$.

The mechanism of formation of a pseudoaneurysm is not completely clear. Ankle arthroscopy is considered as a precipitating cause, because the portals used are in close proximity to neurovascular structures of the ankle. Factors as diabetes, malnutrition, immunosuppression or collagen disorder as Marfan disease or Ehlers-Danlos syndrome compromise the structure of the arterial wall ${ }^{15}$, so they are linked to an increased risk of formation of pseudoaneurysm ${ }^{16}$.

The diagnosis of a pseudoaneurysm is often clinical, a pulsatile mass can be easily identified with palpation of the involved site and it can be confirmed with colorflow duplex ultrasound scan or angiography to clearly define the site and size of the mass. Also an angiography integrated with CT o MRI scan can be helpful ${ }^{17}$. The treatment of a pseudoaneurysm can be surgical or not. Non-surgical treatments include external compression, ultrasound guided compression, ultrasound guid- ed thrombin injection, percutaneous endovascular coil embolisation and percutaneous endovascular stenting. Surgical treatments are ligation- aneurysmectomy and reconstruction with end to end anastomosis or grafting $^{18}$. The best approach is chosen for the specific case observed and it also depends to several factors as the surgeon's experience, or the location of the mass and its relation with surrounding structures ${ }^{19-22}$.

\section{Conclusion}

Formation of a pseudoaneurysm after anterior ankle arthroscopy is extremely rare but it should be suspected when a patient presents abnormal pain, swelling or recurrent hemarthrosis of an ankle, also several weeks after an arthroscopy. To our knowledge 10 cases has been described in literature of formation of pseudoaneurysms and the anterior tibial artery is the vessel mostly involved.

Early identification of a pseudoaneurysm is important in relation with the high risk of rupture of the mass because the structure of its wall is different from the three layers of a natural artery. An accurate anamnesis and physical examination integrated with imaging are the best way to do it. Ultrasound scan is often sufficient to identify the mass. Then prompt treatment is required, surgical or non-surgical, depending from each single case. Pseudoaneurysm of peroneal artery after an anterior ankle arthroscopy was never been described before, so we want to draw attention to it, to avoid iatrogenic lesion performing ankle arthroscopy, considering also this artery as possible site of formation of pseudoaneurysm.

\section{References}

1. Takagi K. The arthroscope. Journal of the Japanese Orthopaedic Association. 1939;14:359.

2. Salgado CJ, Mukherjee D, Quist MA, Cero S. Anterior tibial artery pseudoaneurysm after ankle arthroscopy. Cardiovascular Surgery. 1998;6:604-606.

3. Maffulli N, Del Buono A, Maffulli GD, et al. Isolated anterior talofibular ligament Broström repair for chronic lateral ankle instability: 9-year follow-up. Am J Sports Med. 2013; 41(4):858864.

Table 1. Pseudoaneurysm after anterior ankle arthroscopy ${ }^{7}$.

\begin{tabular}{|c|c|c|c|}
\hline AUTHOR & ARTERY INVOLVED & NUMBER OF CASES & TREATMENT \\
\hline Brimmo et al. ${ }^{9}$ & Anterior tibial & 1 & Embolisation \\
\hline Darwish et al. 5 & Anterior tibial & 1 & Ligation \\
\hline Jacobs et al. ${ }^{13}$ & Anterior tibial & 1 & Embolisation \\
\hline Jang et al. ${ }^{14}$ & Anterior tibial & 1 & Compression \\
\hline Kotwal et al. ${ }^{20}$ & Anterior tibial & 1 & Ligation/vein graft \\
\hline Mariani et al. ${ }^{6}$ & Anterior tibial & 1 & Ligation/vein graft \\
\hline O'Farrell et al. ${ }^{21}$ & Anterior tibial & 1 & Ligation/anastomosis \\
\hline Salgado et al. ${ }^{2}$ & Anterior tibial & 1 & Ligation \\
\hline Yu et al. ${ }^{7}$ & Anterior tibial & 1 & Ligation \\
\hline Kashir et al.22 & Dorsalis pedis & 1 & Ligation/anastomosis \\
\hline
\end{tabular}


4. Ferrann NA, Oliva F, Maffulli N. Ankle instability. Sports Med Arthrosc. 2009;17(2):139-145.

5. Darwish A, Ehsan O, Marynissen H, Al-Khaffaf H. Pseudoaneurysm of the anterior tibial artery after ankle arthroscopy. Arthroscopy. 2004;20:e63-64.

6. Mariani PP, Mancini L, Giorgini TL. Pseudoaneurysm as a complication of ankle arthroscopy. Arthroscopy. 2001;17:400-402.

7. Yu JL, Ho E, Wines AP. Pseudoaneurysms around the foot and the ankle: case report and literature review. Foot and Ankle Surgery. 2013;19:194-198.

8. Padulo J, Oliva F, Frizziero A, Maffilli N. Muscle, Ligaments and Tendon Journal. Basic principles and recommendation in clinical and field science. MLTJ. 2013;4:250-252.

9. Brimmo OA, Parekh SG. Pseudoaneurysm as a complication of ankle arthroscopy. Indian Journal of Orthopaedics. 2010;44 (1):108-111.

10. HASH (0x1039b94) Complications in arthroscopy: the knee and other joints. Arthroscopy. 1986;2:253-258.

11. Dubrueil-Chambardel L. Variations des arteries du pelvis et de member infreiur. Paris Masson et Cie. 1925:191-271.

12. Oliva F, Del Frate D, Ferran NA, Maffilli N. Peroneal tendons subluxation. Sports Med Arthrosc. 2009;17(2):105-111.

13. Palmanovich $\mathrm{E}$, Laver $\mathrm{L}$, Brin $\mathrm{YS}$, et al. Peroneus longus tear and its relation to the peroneal tubercle: A review of the literature. Muscles Ligaments Tendons J. 2012;1(4):153160.

14. Ramavath AL, Cornish JA, Ganapathi M, Williams DT. Missed diagnosis of ankle pseudoaneurysm following ankle arthroscopy: a case report. Cases Journal. 2009;2:162.
15. Dhillon MS, Bali K, Prabhakar S. Differences among mechanoreceptors in healthy and injured anterior cruciate ligaments and their clinical importance. Muscles Ligaments Tendons J. 2012;2(1):38-43.

16. Williams JC, Roberts JW, Yoo BJ. Dorsal pedis artery pseudoaneurysm after lisfranc surgery. Journal of Orthopaedic Trauma. 2010;24:98-101.

17. Jacobs E, Groot D, Das M, Hermus JP. Pseudoaneurysm of the anterior tibial artery after ankle arthroscopy. The Journal of Foot and Ankle Surgery. 2011;50:361-363.

18. Jang EC, Kwak BK, Song KS, Jung HS, Lee JS, Yang JJ. Pseudoaneurysm of the anterior tibial artery after ankle arthroscopy treated with ultrasound-guided compression therapy: a case report. The Journal of Bone and Joint Surgery American Volume. 2008;90-A:2235-2239.

19. Haber LL, Thompson G, Di Domenic L, Groner T, Glaser J. Pseudoaneurysm of the perforating peroneal artery after subtalar joint injury: a case report. Foot \& Ankle International. 2008;29(6):627-629.

20. Kotwal RS, Acharya A, O'Doherty D. Anterior tibial artery pseudoaneurysm in a patient with haemophilia: a complication of ankle arthroscopy. The Journal of Foot and Ankle Surgery. 2007;46(4):314-316.

21. O'Farrell D, Dudeney S, McNally S, Moran R. Pseudoaneurysm formation after ankle arthroscopy. Foot \& Ankle International. 1997;18:578-579.

22. Kashir A, Kiely P, Dar W, et al. Pseudoaneurysm of the dorsalis pedis artery. Injury. 1985;16:424-425. 